\section{Cuantificación de esteatosis hepática no alcohólica por resonancia magnética}

\author{
RODRIGO HERRERA ${ }^{1}$, FRANCISCO PEÑALOZA $A^{1, a, e,}$ \\ CRISTÓBAL ARRIETA ${ }^{1, \mathrm{~b}, \mathrm{f}}$, FLAVIA ZACCONI ${ }^{2, \mathrm{c}, \mathrm{f}}$, \\ VÍCTOR SAAVEDRA ${ }^{3}$, CARLA SAAVEDRA ${ }^{3}$, \\ CECILIA BRAÑES ${ }^{4, g}$, THOMAS HACK ${ }^{4, h}$, SERGIO URIBE ${ }^{1,5,6, d, f}$
}

\section{Quantification of liver fat infiltration by magnetic resonance}

Background: A simple and inexpensive method is required to assess fatty infiltration of the liver non-invasively. Aim: To develop and compare different methods to quantify liver fat by magnetic resonance and compare it against ultrasound. Material and Methods: Three algorithms were implemented: region growing $(R G)$, graph cuts $(G C)$ and hierarchical $(H R)$, all based on the IDEAL method to obtain water and fat images. Using these images, the proton density fat fraction (PDFF) was calculated. The three methods were tested in phantoms with known fat percentages and later on we acquired images from 20 volunteers with an ultrasound diagnosis of fatty liver disease in different stages. For everyone, the PDFF of the nine liver segments was determined. Results: In phantoms, the mean error between the real fat percentage and the value obtained through the three methods was -1,26, -1 and -0,8 for RG, GC and $H R$, respectively. The hierarchical method was more precise and efficient to obtain PDFF. The results in volunteers revealed that ultrasound showed errors categorizing the severity of hepatic steatosis in more than $50 \%$ of volunteers. Conclusions: We developed a tool for magnetic resonance, which allows to quantify fat in the liver. This method is less operator dependent than ultrasound and describes the heterogeneity in the fat distribution along the nine hepatic segments.

(Rev Med Chile 2019; 147: 821-827)

Key words: Fatty Liver; Magnetic Resonance Imaging.

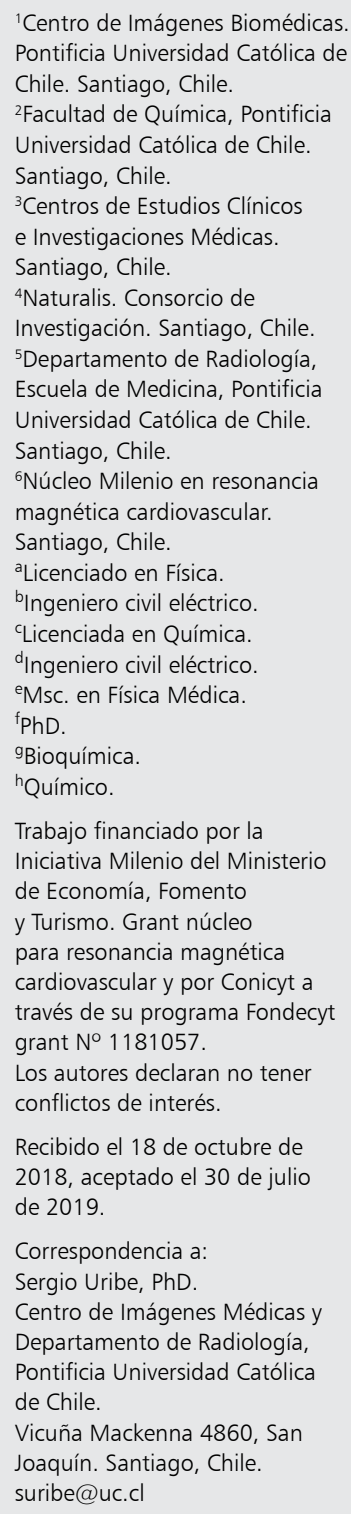

L a esteatosis hepática no alcohólica (EHNA) es la acumulación intracelular de triglicéridos, en ausencia de consumo excesivo de alcohol u otras causas secundarias ${ }^{1}$. Esta patología tiene una prevalencia de 15 a $30 \%$ en adultos ${ }^{2,3}$ y en niños es de 3 a 10\%, llegando a $38 \%$ si son obesos $^{4.5}$, siendo mayor en población hispánica que en caucásicos ${ }^{6}$. La EHNA ha sido relacionado con el síndrome metabólico (SM), la obesidad y la diabetes mellitus, planteándose, incluso, que po- dría tratarse de un factor de riesgo cardiovascular independiente ${ }^{7}$. A nivel continental, Chile solo se posiciona bajo Estados Unidos de Norteamérica en prevalencia de esta enfermedad, y a nivel sudamericano, presenta la prevalencia más elevada junto a Uruguay, Colombia y Venezuela ${ }^{8}$.

Además, la EHNA no solo se relaciona a enfermedades cardiovasculares, sino también a complicaciones y otras enfermedades hepáticas, las cuales son la tercera causa de muerte en Chile 
después de las enfermedades cardiovasculares y tumores malignos ${ }^{9}$. Esta enfermedad es responsable de $20 \%$ de los casos de cirrosis, siendo la tercera causa de años de vida perdidos por discapacidad en hombres ${ }^{10}$. Además, la EHNA puede progresar a esteatohepatitis, grupo con aun mayor probabilidad de evolucionar a cirrosis y finalmente hepatocarcinoma ${ }^{11,12}$.

Aun teniendo en cuenta los datos mencionados, existen vacíos en la historia natural de EHNA y su verdadera prevalencia debido a la carencia de síntomas durante gran parte de su evolución y a la poca sensibilidad de los exámenes de laboratorio para su detección ${ }^{13,14}$. Los datos de la última encuesta nacional de salud muestran que solo $2,8 \%$ de la población reporta el diagnóstico daño hepático. Sin embargo, la analítica realizada a los participantes develó elevaciones de las enzimas hepáticas gamma glutamil transpeptidasa (GGT) y transaminasa glutámico-pirúvica (GPT) en 17,1\% y $15,3 \%$ de los individuos, respectivamente ${ }^{15}$.

Dentro de los exámenes imagenológicos, la ecografía (por su fácil acceso y bajo costo) se ha convertido en la modalidad imagenológica de rutina para evaluar hígado graso. Lamentablemente, tiene baja sensibilidad (20 a 30\%) y especificidad en estadíos tempranos de la enfermedad ${ }^{1,16,17}$, es operador dependiente y tanto la obesidad como la fibrosis hepática disminuyen su rendimiento.

Otros métodos, como la tomografía computada (TC) y la resonancia magnética (RM), también pueden diagnosticar EHNA. Sin embargo, la TC tampoco detecta estadíos tempranos y agrega la exposición a radiación. En el caso de la RM, existen dos posibilidades para cuantificar el EHNA, la espectroscopía y los métodos de imágenes. La espectroscopía tiene alta sensibilidad, pero solo cuantifica la grasa contenida en una zona local del hígado, obteniendo la señal de un solo voxel de aproximadamente $3 \times 3 \times 3 \mathrm{~cm}$, por lo que no describe la heterogeneidad en la distribución de la grasa en el hígado ${ }^{18}$ y es, además, una técnica muy sensible al movimiento del paciente y a las imperfecciones del sistema de RM.

Debido a las limitaciones mencionadas de espectroscopía, se ha desarrollado la técnica de imagen densidad protónica de fracción grasa (PDFF por sus siglas en inglés de proton density fat fraction), que permite cuantificar la grasa hepática en todo el hígado. A través de esta técnica es posible obtener un mapa cuantitativo de porcentaje en todo el hígado. Adicionalmente, sus resultados han sido validados con histología, resultando ser una medida precisa y robusta ${ }^{19-21}$.

Actualmente han sido propuestos distintos métodos para calcular el PDFF, como los métodos Region Growing Ideal, Graph Cuts y Hierarchical IDEAL, entre otros ${ }^{22,23}$. Sin embargo, estos métodos no se encuentran disponibles ampliamente en la mayoría de los resonadores y tampoco está claro cuál de los métodos es más robusto y preciso.

En este trabajo desarrollamos una metodología en que, a partir de las imágenes de cualquier resonador magnético, podemos reconstruir el PDFF, utilizando cualquiera de los métodos antes mencionados. A partir de esto, realizamos una comparación uno a uno de los 3 métodos en emulsiones de agua y grasa con distintos porcentajes de grasa conocido y en pacientes previamente diagnosticados con hígado graso por ecografía.

\section{Materiales y Métodos}

\section{Cálculo de PDFF}

A partir de imágenes multieco de resonancia magnética y de métodos basados en el algoritmo de IDEAL se obtuvo una imagen de agua $\left(\rho_{\text {water }}\right)$ y una de grasa $\left(\rho_{\text {fat }}\right)$, a partir de las cuales se calculó el PDFF como:

$$
\operatorname{PDFF}=\frac{\left|\rho_{\text {fat }}\right|}{\left|\rho_{\text {fat }}\right|+\left|\rho_{\text {water }}\right|}
$$

En un software computacional implementamos 3 métodos para obtener el PDFF que se compararán: Region Growing (RG), Graph Cuts (GC) y Hierarchical (HR).

\section{Pruebas en fantomas de emulsiones de agua y grasa}

Los 3 métodos fueron testeados en 8 fantomas consistentes en tubos Falcon que contenían emulsiones de agua y grasa con distintos porcentajes grasa igual a $0 \%, 5 \%, 10 \%, 15 \%, 20 \%, 25 \%, 30 \%$ y $40 \%$.

\section{Pruebas en pacientes con hígado graso}

Posteriormente, se obtuvieron imágenes de 20 voluntarios sanos ( $\sin$ antecedente de alcoholismo u otras causas de esteatosis) con diagnóstico previo de hígado graso por ecografía (utilizando índice 
hepatorrenal). Los pacientes fueron cinco hombres y quince mujeres, entre 33 y 69 años de edad.

En pacientes, el diagnóstico ecográfico consistió en una escala semicualitativa donde un médico radiólogo clasificó al paciente como sano o con hígado graso, en cuyo caso, clasificamos también el grado de compromiso en leve, moderado o severo.

Los resultados de la ecografía se compararon con los resultados del PDFF. Para ello, clasificamos el grado de esteatosis con el valor PDFF de acuerdo a Tang et $\mathrm{al}^{21}$.

- Sin hígado graso: $\leq 6,4 \%$ grasa.

- Leve: $>6,4 \%$ grasa.

- Moderado: $>17,4 \%$ grasa.

- Severo: $>22,1 \%$ grasa.

El proyecto fue aprobado por el Comité de Ética de la Pontificia Universidad Católica de Chile y se obtuvo consentimiento informado de todos los participantes.

\section{Protocolo de resonancia magnética}

Las pruebas en fantomas reales, voluntarios $y$ pacientes fueron realizadas en un resonador Philips Achieva 1,5T (Philips, Best, Países Bajos). Los parámetros de adquisición de resonancia magnética se muestran en la Tabla 1.

\section{Análisis de datos}

Evaluamos el comportamiento de los 3 métodos de cuantificación de PDFF comparando la diferencia entre el valor medido con el valor real

\section{Tabla 1. Parámetros de adquisición para secuencia de resonancia magnética}

\begin{tabular}{|ll|}
\hline Parámetro & 2D \\
\hline Resolución $(\mathrm{mm})$ & $1,5 \times 1,5$ \\
\hline Espesor $(\mathrm{mm})$ & 9 \\
\hline TR $(\mathrm{ms})$ & 30 \\
\hline Ángulo de excitación $\left(^{\circ}\right)$ & 8 \\
\hline Número de cortes & $15-24$ \\
\hline Número de ecos & 6 \\
\hline TE (ms)/ $\triangle$ TE (ms) & $1,3 / 2,1$ \\
\hline Orientación & Derecha izquierda-anteroposterior \\
\hline Campo de visión (mm) & Dependiente del sujeto \\
\hline Matriz de adquisición & Dependiente del FOV \\
\hline
\end{tabular}

TR: tiempo de repetición, TE: tiempo de eco, $\triangle T E$ : tiempo entre ecos, FOV: campo de visión, TFE: factor turbo. de porcentaje de grasa en las emulsiones de grasa y agua. El valor medido correspondió al promedio de 3 mediciones realizadas a distintos niveles de cada emulsión. Luego, se escogió el método más preciso y eficiente para la medición de la PDFF en pacientes.

En las imágenes de pacientes dibujamos regiones de interés (ROI) en los nueve segmentos hepáticos de Couinaud para obtener el promedio de PDFF y la desviación estándar (SD). Las ROI se dibujaron en la imagen de agua para evitar vasos sanguíneos y luego fueron trasladadas al mapa de fracción de grasa.

\section{Resultados}

Los resultados de los 3 algoritmos con 8 concentraciones de grasa se muestran en la Tabla 2.

El error promedio entre el valor analítico y el obtenido por los distintos métodos fue de $-1,26$, -1 y -0,8 para el método RG GC, y Hierarchical, respectivamente. Se puede apreciar, además, que obtienen una excelente concordancia con los datos reales de los fantomas. En resumen, es posible obtener resultados de cuantificación del mapa de PDFF con errores máximos de entre $2 \%$ y $3 \%$. Se puede apreciar que el método Hierarchical IDEAL es el que tiene mayor precisión y eficiencia para el cálculo de PDFF.

\section{Resultados pacientes}

En la Figura 1, se muestra un ejemplo de la PDFF obtenida en un caso con esteatosis leve, moderada y severa de acuerdo al PDFF. La Tabla 3 describe la demografía de los pacientes, el diagnóstico de referencia realizado por ecografía, los valores de PDFF y la clasificación en leve, moderado y severo de acuerdo a Tang et al. Un paciente estaba en el rango de peso saludable (índice de masa corporal [IMC] entre 18,5 y 24,9), siete con sobrepeso (IMC entre 25 y 29,9 ) y doce obesos (IMC por encima de 30).

De acuerdo a lo observado en la Tabla 3, vemos que la ecografía sobreestima o subestima el nivel grasa hepática:

La ecografía detectó correctamente solo a 3 de 9 sujetos con esteatosis leve, el resto (junto a un sujeto sano), fueron catalogados como moderado. De los 2 casos de esteatosis moderada, diagnosticó solo a 1 y de 8 sujetos con esteatosis severa, catalogó correctamente solo a 2 . 
Tabla 2. Resultados de la cuantificación del PDFF con distintos métodos en distintas emulsiones de agua y grasa

\begin{tabular}{|crrr|}
\hline \% de grasa real & Region Growing & Graph Cuts & Hierarchical \\
\hline 0 & $2,18 \pm 1,15$ & $1,61 \pm 0,83$ & $2,20 \pm 2,05$ \\
\hline 5 & $5,27 \pm 1,83$ & $4,76 \pm 0,96$ & $5,37 \pm 2,43$ \\
\hline 10 & $10,21 \pm 1,56$ & $10,47 \pm 1,04$ & $10,15 \pm 2,54$ \\
\hline 15 & $16,29 \pm 1,14$ & $15,58 \pm 1,32$ & $15,41 \pm 2,27$ \\
\hline 20 & $21,98 \pm 1,18$ & $22,14 \pm 0,78$ & $21,93 \pm 2,98$ \\
\hline 30 & $27,40 \pm 1,14$ & $27,46 \pm 0,86$ & $26,43 \pm 2,22$ \\
\hline 0 & $30,03 \pm 0,99$ & $30,00 \pm 0,74$ & $29,55 \pm 2,89$ \\
\hline
\end{tabular}

Tabla 3. Demografía de voluntarios, diagnóstico de referencia ecográfico, la clasificación de hígado graso según Tang et al. y el valor promedio y rango de PDFF

\begin{tabular}{|c|c|c|c|c|c|c|c|c|}
\hline Paciente & Edad & $\begin{array}{l}\text { Peso } \\
(\mathbf{k g})\end{array}$ & $\begin{array}{l}\text { Altura } \\
\text { (m) }\end{array}$ & IMC & Ecografía & $\begin{array}{l}\text { PDFF } \\
\text { (Tang) }\end{array}$ & \multicolumn{2}{|c|}{$\begin{array}{c}\text { PDFF promedio y } \\
\text { rango }\end{array}$} \\
\hline 1 & 34 & 107 & 1,8 & 33,02 & Moderado & Severo & 25,11 & {$[13,10-30,70]$} \\
\hline 2 & 33 & 100 & 1,79 & 31,21 & Moderado & Severo & 19,23 & {$[17,45-21,27]$} \\
\hline 3 & 54 & 100 & 1,83 & 29,86 & Moderado & Moderado & 17,49 & {$[14,11-20,36]$} \\
\hline 4 & 61 & 110 & 1,75 & 35,92 & Moderado & Leve & 11,82 & {$[8,76-14,40]$} \\
\hline 5 & 55 & 104 & 1,69 & 36,41 & Moderado & Severo & 25,67 & {$[20,13-28,5]$} \\
\hline 6 & 48 & 97 & 1,56 & 39,86 & Moderado & Severo & 21,72 & {$[17,9-25,26]$} \\
\hline 7 & 42 & 63 & 1,7 & 21,8 & Leve & Leve & 15,88 & {$[14,3-16,72]$} \\
\hline 8 & 49 & 95 & 1,6 & 37,11 & Leve & Leve & 12,65 & {$[11,28-15,14]$} \\
\hline 9 & 53 & 76 & 1,53 & 32,47 & Moderado & Leve & 13,52 & {$[7,26-18,05]$} \\
\hline 10 & 34 & 85 & 1,69 & 29,76 & Leve & Leve & 5,98 & {$[4,34-7,24]$} \\
\hline 11 & 59 & 58 & 1,46 & 27,21 & Moderado & Leve & 5,21 & {$[3,86-6,24]$} \\
\hline 12 & 63 & 105 & 1,78 & 33,14 & Severo & Severo & 25,11 & {$[24,25-26,57]$} \\
\hline 13 & 56 & 68 & 1,58 & 27,24 & Leve & Leve & 10,31 & {$[8,94-12,75]$} \\
\hline 14 & 50 & 83 & 1,64 & 30,86 & Severo & Severo & 29,81 & {$[27,54-31,9]$} \\
\hline 15 & 55 & 71 & 1,63 & 26,72 & Moderado & No & 6,93 & {$[6,11-7,68]$} \\
\hline 16 & 62 & 87 & 1,64 & 32,35 & Moderado & No & 5,68 & {$[3,97-7,15]$} \\
\hline 17 & 69 & 69 & 1,59 & 27,29 & Moderado & Leve & 10,64 & {$[7,6-14,71]$} \\
\hline 18 & 54 & 75 & 1,6 & 29,30 & Moderado & Severo & 19,22 & {$[15,44-22,71]$} \\
\hline 19 & 60 & 80 & 1,6 & 31,25 & Moderado & Severo & 17,9 & {$[14,88-20,17]$} \\
\hline 20 & 46 & 91 & 1,6 & 35,55 & Moderado & Leve & 7,9 & {$[6,17-9,90]$} \\
\hline
\end{tabular}




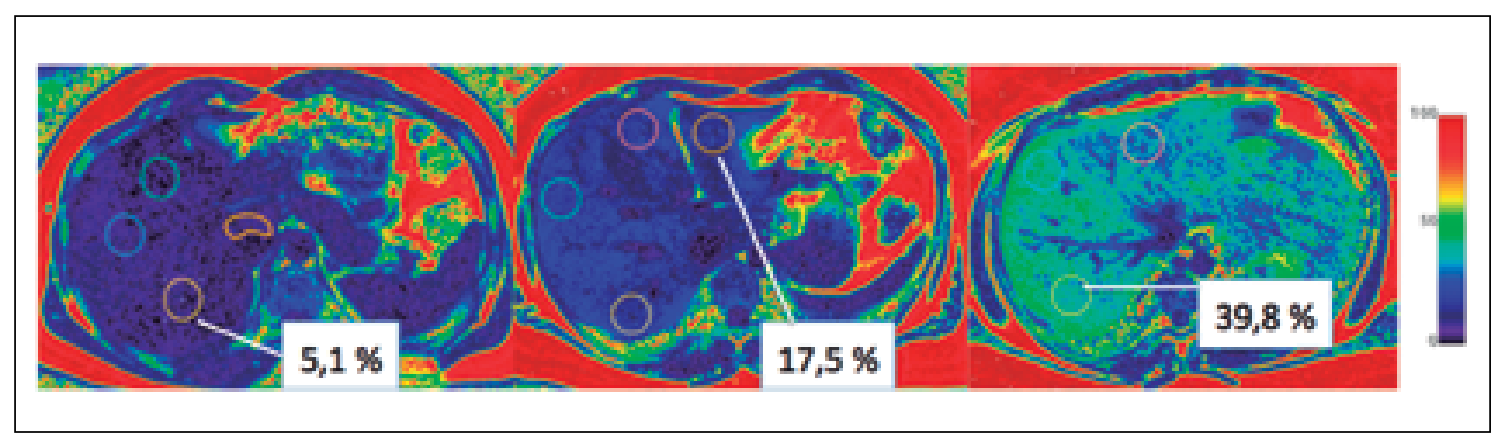

Figura 1. Imágenes obtenidas a través de software. De izquierda a derecha se presentan individuos con esteatosis leve, moderada y severa, respectivamente. Circunferencias representan los ROI en un segmento de Couinaud. Se muestra en cada figura el porcentaje de grasa en un ROI determinado.

\section{Discusión}

A partir de los experimentos en fantomas podemos concluir que hemos implementado distintos algoritmos para la cuantificación de PDFF, como los métodos de Region Growing, Graph Cuts y Hierarchical, los cuales nos permiten obtener una cuantificación precisa del PDFF.

Los sujetos de estudio constituyeron un grupo de muestra diverso, que incluyó sujetos con diferentes IMC y niveles de esteatosis hepática. Debido a que la prevalencia de esteatosis en sujetos con IMC normal es baja y a que los sujetos reclutados fueron diagnosticados por ecografía, pesquisamos solo a 1 individuo con estas características. La prevalencia de EHNA en personas con IMC normal va de 7,2 a 12,6\%, dependiendo del estudio ${ }^{24-26}$. Además, la baja sensibilidad de la ecografía en estadíos leves, hace que la pesquisa de EHNA en personas con IMC normal sea difícil. Esperamos poder generar datos sobre esto en un futuro cercano en una muestra mayor de individuos.

A través de este estudio hemos demostrado que la PDFF es un método cuantitativo y más preciso que la ecografía (hoy el método estándar) para el diagnóstico de hígado graso. Además, este método es capaz de evaluar el órgano en su totalidad, superando en este aspecto a la espectroscopía en RM.

La experiencia aportada por la Salud Pública nos muestra que los individuos con etapas preclínicas de enfermedad son los que más se benefician de intervenciones en salud. En este contexto, quedará pendiente a futuro la evalua- ción costo-beneficio del diagnóstico fidedigno de esteatosis, tomando en cuenta el incremento continuo de gastos derivados de enfermedades cardiovasculares y relacionadas al daño hepático.

Desde un punto de vista de Salud Pública, cabe preguntarse en qué personas sería adecuado realizar este examen, o en qué momento sería necesario el control una vez que están diagnosticados. Se necesitarán estudios longitudinales para responder estas preguntas y, en ese sentido, una las principales aplicaciones de esta técnica es el seguimiento de los pacientes. A diferencia de la ecografía, con esta herramienta podemos constatar cambios en la concentración de grasa, evaluando la progresión de la enfermedad a medida que aumenta la proporción de grasa. La reproducibilidad, entonces, es esencial para el cumplimiento de dicho objetivo. Del punto de vista técnico, las distintas técnicas presentadas pueden evaluar imágenes de distintos resonadores magnéticos, por lo que, el uso de esta técnica podría realizarse en casi cualquier resonador magnético. Se ha mostrado también que técnicas similares a las descritas en este trabajo tienen una gran reproducibilidad y los valores obtenidos son independientes de operador, centro, y marca de resonador magnético ${ }^{27}$.

En conclusión, hemos desarrollado un método que hace viable la aplicación del cálculo de la PDFF en imágenes de resonancia magnética, el cual provee un diagnóstico preciso de la cantidad de grasa hepática y ayudará a resolver la brecha existente en el conocimiento actual sobre su historia natural y real magnitud en términos de prevalencia. 


\section{Referencias}

1. Graffigna M, Catoira N, Soutelo J, Azpelicueta A, Berg G, Perel C, et al. Diagnóstico de esteatosis hepática por métodos clínicos, bioquímicos y por imágenes. Rev Argent Endocrinol Metab 2017; 54 (1): 37-46.

2. Younossi ZM, Koenig AB, Abdelatif D, Fazel Y, Henry L, Wymer M. Global epidemiology of nonalcoholic fatty liver disease-Meta-analytic assessment of prevalence, incidence, and outcomes. Hepatol Commun 2016; 64 (1): 73-84

3. Mikolasevic I, Orlic L, Franjic N, Hauser G, Stimac D, Milic S. Transient elastography (FibroScan $((\mathrm{R}))$ ) with controlled attenuation parameter in the assessment of liver steatosis and fibrosis in patients with nonalcoholic fatty liver disease - Where do we stand? World J Gastroenterol 2016; 22 (32): 7236-51.

4. Anderson EL, Howe LD, Jones HE, Higgins JP, Lawlor DA, Fraser A. The Prevalence of Non-Alcoholic Fatty Liver Disease in Children and Adolescents: A Systematic Review and Meta-Analysis. PLoS One 2015; 10 (10): e0140908.

5. Mann JP, Valenti L, Scorletti E, Byrne CD, Nobili V. Nonalcoholic Fatty Liver Disease in Children. Semin Liver Dis 2018; 38 (1): 1-13.

6. Schwimmer JB, McGreal N, Deutsch R, Finegold MJ, Lavine JE. Influence of gender, race, and ethnicity on suspected fatty liver in obese adolescents. Pediatrics 2005; 115 (5): e561-5.

7. National Institute for Health and Care Excellence (2016) Non-Alcoholic Fatty Liver Disease: Assessment and Management (NICE Guideline, No. 49) . Disponible en: https://www.nice.org.uk/guidance/ng49. [Consultado el 2 de septiembre de 2018].

8. Younossi Z, Anstee QM, Marietti M, Hardy T, Henry L, Eslam M, et al. Global burden of NAFLD and NASH: trends, predictions, risk factors and prevention. Nat Rev Gastroenterol Hepatol 2017; 15: 11-20.

9. Departamento de Estadísticas e Información en Salud. Gobierno de Chile, Ministerio de Salud. Indicadores Básicos de Salud 2015. Disponible en: http://www.deis. cl/wp-content/uploads/2018/05/IBS-2015.pdf. [Consultado el 2 de septiembre de 2018].

10. Alonso FT, Garmendia ML, Aguirre M, Searle J. [Mortality trend from liver cirrhosis in Chile from 1990 to 2007]. Rev Med Chile 2010; 138 (10): 1253-8.

11. Bugianesi E, Leone N, Vanni E, Marchesini G, Brunello F, Carucci P, et al. Expanding the natural history of nonalcoholic steatohepatitis: from cryptogenic cirrhosis to hepatocellular carcinoma. Gastroenterology 2002; 123 (1): 134-40.
12. Caldwell SH, Crespo DM. The spectrum expanded: cryptogenic cirrhosis and the natural history of non-alcoholic fatty liver disease. J Hepatol 2004; 40 (4): 57884.

13. Chalasani N, Younossi Z, Lavine JE, Diehl AM, Brunt $\mathrm{EM}$, Cusi $\mathrm{K}$, et al. The diagnosis and management of non-alcoholic fatty liver disease: practice Guideline by the American Association for the Study of Liver Diseases, American College of Gastroenterology, and the American Gastroenterological Association. Hepatol Commun 2012; 55 (6): 2005-23.

14. Wilman HR, Kelly M, Garratt S, Matthews PM, Milanesi $\mathrm{M}$, Herlihy A, et al. Characterisation of liver fat in the UK Biobank cohort. PLoS One 2017; 12 (2): e0172921.

15. Encuesta Nacional de Salud 2009-2010. Ministerio de Salud, Gobierno de Chile. Sección V.2.11, Daño hepático crónico; p. 302-303, 307.Disponible en: http:// www.minsal.cl/portal/url/item/bcb03d7bc28b64dfe040010165012d23.pdf [Consultado el 2 de septiembre de 2018].

16. Hashimoto E, Taniai M, Tokushige K. Characteristics and diagnosis of NAFLD/NASH. JGH Open 2013; 28 (4): 64-70.

17. Lee DH. Imaging evaluation of non-alcoholic fatty liver disease: focused on quantification. Clin Mol Hepatol 2017; 23 (4): 290-301.

18. Idilman IS, Ozdeniz I, Karcaaltincaba M. Hepatic Steatosis: Etiology, Patterns, and Quantification. Semin Ultrasound CT MR 2016; 37 (6): 501-10.

19. Tang A, Tan J, Sun M, Hamilton G, Bydder M, Wolfson $\mathrm{T}$, et al. Nonalcoholic fatty liver disease: MR imaging of liver proton density fat fraction to assess hepatic steatosis. Radiology 2013; 267 (2): 422-31.

20. Idilman IS, Aniktar H, Idilman R, Kabacam G, Savas B, Elhan A, et al. Hepatic steatosis: quantification by proton density fat fraction with MR imaging versus liver biopsy. Radiology 2013; 267 (3): 767-75.

21. Tang A, Desai A, Hamilton G, Wolfson T, Gamst A, Lam J, et al. Accuracy of MR imaging-estimated proton density fat fraction for classification of dichotomized histologic steatosis grades in nonalcoholic fatty liver disease. Radiology 2015; 274 (2): 416-25.

22. Tsao J, Jiang Y. Hierarchical IDEAL: fast, robust, and multiresolution separation of multiple chemical species from multiple echo times. Magn Reson Med 2013; 70 (1): 155-9.

23. Hernando D, Kellman P, Haldar JP, Liang ZP. Robust water/fat separation in the presence of large field inhomogeneities using a graph cut algorithm. Magn Reson Med 2010; 63 (1): 79-90.

24. Kwon YM, Oh SW, Hwang SS, Lee C, Kwon H, Chung 
GE. Association of nonalcoholic fatty liver disease with components of metabolic syndrome according to body mass index in Korean adults. Am J Gastroenterol 2012; 107: 1852-8.

25. Xu C, Yu C, Ma H, Xu L, Miao M, Li Y. Prevalence and risk factors for the development of nonalcoholic fatty liver disease in a nonobese Chinese population: the Zhejiang Zhenhai Study. Am J Gastroenterol 2013; 108: 1299-304
26. Younossi ZM, Stepanova M, Negro F, Hallaji S, Younossi Y, Lam B, et al. Nonalcoholic fatty liver disease in lean individuals in the United States. Medicine (Baltimore) 2012; 91: 319-27.

27. Kang GH, Cruite I, Shiehmorteza M, Wolfson T, Gamst, AC, Hamilton G, et al. Reproducibility of MRI-determined proton density fat fraction across two different MR scanner platforms. J. Magn. Reson. Imaging 2011; 34: 928-34. doi:10.1002/jmri.22701. 